\title{
Ventricular septal defect: (not) another epidemic?
}

\author{
David F. Dickinson \\ Yorksbire Heart Centre, Leeds General Infirmary, Leeds, UK
}

$\mathrm{E}$ PIDEMIOlOgical Studies OF CONGENITAL heart disease performed in the 1960's using traditional clinical methods showed the prevalence of ventricular septal defect to be around 3 cases per 1000 live births. ${ }^{1}$ A relatively small increase in the number of cases reported in the late 1970's and early 1980's led to suggestions of an 'epidemic'. Most would now agree that this increased ascertainment, mainly of small defects, was the result of the development of high resolution cross sectional echocardiography, ${ }^{2}$ probably coupled with the trend for referral of asymptomatic patients with soft murmurs for definitive diagnosis at a younger age.

Recently published papers raise similar questions concerning a new 'epidemic'. In 1992, Hiraishi and colleagues ${ }^{3}$ performed colour Doppler ultrasound on 1028 consecutive term infants and identified 26 with defects in the ventricular septum (21 having defects in the apical trabecular septum), a prevalence of 25.2 per 1000 live births.

More recently, and again using colour Doppler echocardiography prospectively on 1053 consecutive newborn infants, Roguin and colleagues ${ }^{4}$ identified 56 with isolated defects in the apical trabecular part of the ventricular septum, a prevalence of 53.2 per 1000 live births. This figure is about 15 to 20 times greater than that recorded in most previous studies, where ascertainment of cases was by clinical methods. In the accompanying paper, ${ }^{3}$ again using colour Doppler, the same group have found 103 ventricular septal defects in 7696 consecutive live births, giving a prevalence of

Correspondence to: David F. Dickinson, Yorkshire Heart Centre, Leeds Genera Infirmary (Efloor), Great George Street, Leeds LSI 3EX, UK. Tel: 0113432799 ; Fax: 01133925784

13.4 per 1000 live births. The dates during which data were collected suggest that the second study was an extension of the first. If so, the initial group of 1053 patients are presumably part of the larger group of 7696 patients. If this is indeed the case, then 56 defects were identified in the first 1053 patients studied (prevalence 53.2 per 1000), but only 47 were identified among the next 6643 (prevalence 7.1 per 1000). This represents a very significant change during the relatively short period of 19 months. Even if the two groups are completely separate, the difference in prevalence is very large when one considers that they were studied by the same investigators in the same institution using the same technique.

In their first paper the authors speculate on the reasons for the very high prevalence of ventricular septal defect. Not unreasonably, they conclude that the use of colour Doppler permitted identification of many defects which would not have been detected on clinical grounds alone. They have not addressed at any point, however the question of why the prevalance of ventricular septal defect is so dramatically lower in the second study. Although the authors appear to have taken every care to avoid false positive diagnosis, the devil's advocate would argue that the most likely explanation is a subtle change in ultrasonic technique rather than an unidentified teratogen affecting a small group of patients during a relatively short period of time, as the authors suggest tentatively in their original paper.

Studies of the chick embryo have shown that the apical trabecular septum has many communicating interventricular channels which are progressively obliterated as the embryo matures. ${ }^{6}$ As long ago as 1970 , Mitchell and colleagues ${ }^{7}$ suggested that, in man, the time for closure of the ventricular septum may extend beyond the fifth post conceptual week, possibly even into post natal life. These recent papers describing ultrasonic findings tend to 
endorse that view. It will be fascinating to discover the prevalence of ventricular septal defects at 16 to 20 weeks gestation if, and when, fetal cardiac ultrasonic technology provides the resolution of detail now possible in the neonatal period.

What are the implications of these findings for clinical practice?

Whatever the precise prevalence of ventricular septal defect at birth, it is clear that if time, expertise and resources are devoted to screening newborns using colour Doppler ultrasound, a large number of unsuspected ventricular septal defects will be identified, together with presumably equally large numbers of infants with shunting at atrial and great arterial levels through the oval fossa and the arterial duct. The resource implications of such an exercise are considerable. It is probably unlikely that many centres would wish to screen entire populations in this way. As high resolution colour Doppler equipment becomes more widely available, nonetheless, it is probable that many of these defects will be identified by chance in infants with a variety of transient cardiorespiratory problems who are studied in neonatal intensive care units. The identification of a 'hole in the heart' causes considerable anxiety for families, and it will be necessary to have in place appropriate facilities for counselling and follow up. Clearly this technical advance, whilst possibly providing new insights into the development of the heart and the epidemiology of congenital heart disease, also has the potential to create unnecessary anxiety among families. It may also significantly increase workload for paediatric cardiologists if not managed with care and discretion.

\section{References}

1. Hoffman JIE, Christianson R. Congenital heart disease in a cohort of 19502 births with long term follow-up. Am J Cardiol 1966; 18: 713-723.

2. Martin GR, Perry LW, Ferencz C. Increased prevalence of ventricular septal defect: epidemic or improved diagnosis? Pediatrics 1989; 83: 200-203

3. Hiraishi S, Agata $Y$, Nowatari $M$, Oguchi $K$, Hirota $H$. Incidence and natural course of trabecular ventricular septal defect: two dimensional echocardiography and color Doppler flow imaging study. J Pediatrics 1992; 120: 409-415.

4. Roguin N, Zhong-Dong Du, Barak M. Nasser N, Hershkowitz S, Milgram E. J Am Coll Cardiol 1995; 26: 1545-1548.

5. Zhong-Dong Du, Roguin N, Xing-Jian Wu. Spontaneous closure of muscular ventricular septal defect identified by echocardiography in neonates. Cardiol Young 1998; 8 (4): 500-505

6. Ben-Shachar G, Arcilla RA, Lucas RV, Manasek FJ. Ventricular trabeculations in the chick embryo herat and their contribution to ventricular muscular septal development. Circulation Research 1985; 57: 759-766.

7. Mitchell SC Berendes HW, Clark WM Jnr. The normal closure of the ventricular septum. Am Heart J 1970; 80: 432-433. 